\title{
La importancia de la uniformización del formato de objetos didácticos en estudios interuniversitarios
}

\author{
Isidoro Iván Cuesta Segura \\ Universidad de Burgos (España) \\ iicuesta@ubu.es \\ Jesús Manuel Alegre CALDERón \\ Universidad de Burgos (España) \\ jalegre@ubu.es
}

\section{Resumen}

En la actualidad, los objetos didácticos son un pilar fundamental en la educación superior. Resulta fundamental establecer unos criterios de uniformización de sus formatos que sean comunes a todas las asignaturas que conforman un plan de estudios, en especial si se trata de estudios interuniversitarios, para conseguir maximizar la empleabilidad de dichos recursos del aprendizaje.

Palabras clave: Objetos didácticos; uniformización del formato; estudios interuniversitarios

\section{The importance of standardization of learning object format in interuniversity studies}

\begin{abstract}
Currently, learning objects are a mainstay in higher education. It is essential to establish uniform criteria of formats that are common to all the subjects included in a study plan, especially in interuniversity studies, in order to maximize the employability of said learning resources.
\end{abstract}

Key Words: Learning objects; standardization of format; interuniversity studies

Referencia normalizada:

Cuesta Segura, I. I. y Alegre Calderón, J. M. (2013) La importancia de la uniformización del formato de objetos didácticos en estudios interuniversitarios. Historia y Comunicación Social. Vol. 18. № Especial Noviembre. Págs. 725-730.

Sumario: 1. Introducción. 2. Metodología e implantación. 2.1. Actuación 1. Recopilación e identificación de los objetos didácticos empleados en el master 2.2. Actuación 2. Uniformización del formato de los objetos didácticos que se utilizarán en el master 2.3. Actuación 3. Conclusiones.

\section{Introducción}

Los objetos didácticos o learning objects son un pilar fundamental en la actualidad en la educación superior para la aplicación de las TIC. Dichos objetos consisten básicamente en fragmentar los contenidos educativos en unidades modulares independientes que pueden ser reutilizadas por diferentes aplicaciones y en diversos 
entornos, David Wiley (2000) y Salvador Sanchez Alonso (2005). A menudo reciben el nombre de objetos didácticos reutilizables y se han convertido en el elemento central del diseño de contenidos docentes en los entornos de trabajo virtual basados en Moodle que emplean la mayoría de las universidades españolas.

Durante la etapa de gestación y definición de los objetos didácticos que conforman una determinada asignatura el formato empleado por el docente es fundamental. Si se desea maximizar su empleabilidad por parte del estudiante es aconsejable poner especial atención al formato de cada uno de ellos para que todos tengan el mismo aspecto, ya que de lo contrario la comprensión por parte del alumnado podría verse comprometida significativamente, Miguel Ángel Sicilia (2005).

El gran inconveniente para lograr esta meta de manera global en un plan de estudios cualquiera radica en la falta de coordinación entre los docentes que imparten las asignaturas de ese plan de estudios a la hora de definir un formato único para los objetos didácticos de todas las asignaturas. Lo que desemboca en diferentes formatos en función de la asignatura que se analice. Si a este hecho le añadimos que los docentes pueden llegar a pertenecer a distintos centros o incluso distintas universidades es posible que los objetos didácticos empleados no lleguen a satisfacer por completo las expectativas del estudiante referentes a este tema.

Este podría ser el caso del Master Interuniversitario de Integridad y Durabilidad de Materiales, Componentes y Estructuras que se imparte en las Universidades de Oviedo (UNIOVI), Cantabria (UNICAN) y Burgos (UBU), donde los docentes involucrados en la impartición del Master han creado por separado los objetos didácticos de cada una de las asignaturas que conforman el título. El presente trabajo se va a centrar en la mejora de la uniformización del formato de los objetos didácticos de dicho Master, aunque todos los aspectos planteados serían extrapolables a cualquier otra titulación o estudio interuniversitario.

En consecuencia, es fundamental la identificación de unos criterios básicos para la uniformización del formato de los objetos didácticos de todas las asignaturas que componen el Master Interuniversitario en Integridad y Durabilidad de Materiales, Componentes y Estructuras. Para ello hay que trabajar y profundizar en los siguientes aspectos:

- Homogeneizar el formato de los objetos didácticos generados por los docentes de las tres universidades involucradas en el Master.

- Establecer una o varias plantillas para la entrega de trabajos por parte de los estudiantes.

- Definir una plantilla válida para la realización del Trabajo Fin de Master (TFM). 


\section{Metodología e implantación}

Para lograr conseguir los aspectos planteados en este trabajo se proponen una serie de actuaciones o tareas. La duración de cada una de las actuaciones se adaptará a los avances conseguidos, logrando finalmente su total implantación. La coordinación, seguimiento y evaluación del nivel de ejecución de estas tareas es aconsejable que se lleve a cabo mediante reuniones periódicas entre los coordinadores del Master de cada universidad.

2.1 Actuación 1. Recopilación e identificación de los objetos didácticos empleados en el master

Debido a que en la actualidad el Master Interuniversitario en Integridad y Durabilidad de Materiales, Componentes y Estructuras se encuentra ya implantado de manera satisfactoria en las Universidades de Oviedo, Cantabria y Burgos es posible afirmar que prácticamente el $100 \%$ de los objetos didácticos empleados se encuentran generados para todas las asignatura que conforman el Master. Esta tarea ha sido realizada durante los últimos años por el docente responsable de cada una de las asignaturas, el cuál pertenecerá a una u otra universidad en función de la asignatura de que se trate (Figura 1).

Figura 1. Relación de asignaturas del Master

\begin{tabular}{|l|c|}
\hline \multicolumn{1}{|c|}{ Asignatura del Master } & Universidad responsable \\
\hline Teoría de la Elasticidad y Plasticidad & U. Burgos \\
\hline Simulación numérica de materiales, componentes y estructuras & U. Burgos \\
\hline Materiales estructurales & U. Oviedo \\
\hline Mecánica de la fractura & U. Oviedo \\
\hline Fatiga, corrosión bajo tensión y fluencia & U. Cantabria \\
\hline Procedimientos de evaluación de la integridad estructural & U. Cantabria \\
\hline Soldadura y tecnologías de unión & U. Oviedo \\
\hline Corrosión, oxidación, desgaste y protección & U. Burgos \\
\hline Análisis de fallos & U. Cantabria \\
\hline Técnicas de inspección y ensayos no destructivos & U. Oviedo \\
\hline Seminario especializado I & Todas \\
\hline Seminario especializado II & Todas \\
\hline TFM & Todas \\
\hline
\end{tabular}

Fuente: Elaboración propia

Por tanto esta primera actuación consistirá en recopilar e identificar dichos objetos didácticos, así como el formato empleado en cada uno de ellos. Dado que el Master se 
imparte en su gran mayoría por videoconferencia la mayoría de los objetos didácticos corresponden a presentaciones en formato PowerPoint. Para ello se involucrará a los docentes responsables de cada una de las asignaturas del Master con el objetivo de uniformizar el formato empleado en las asignaturas. A modo de ejemplo, la Figura 2 muestra una diapositiva de uno de estos objetos correspondiente a la asignatura Simulación numérica de materiales, componentes y estructuras, la cual se encuentra sin uniformizar el formato.

Figura 2. Ejemplo de objeto didáctico sin uniformizar.

\section{Comparación de resultados}

- $\mathrm{MFEL} \rightarrow \mathrm{J}$ con roseta de elementos singulares

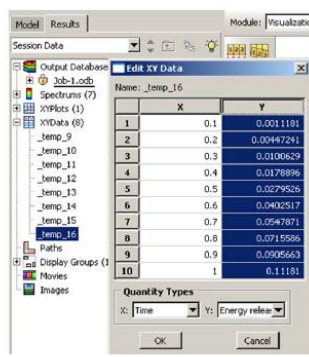

$$
J=K_{I}^{2} / E \Rightarrow K_{I}=\sqrt{J \cdot E}
$$
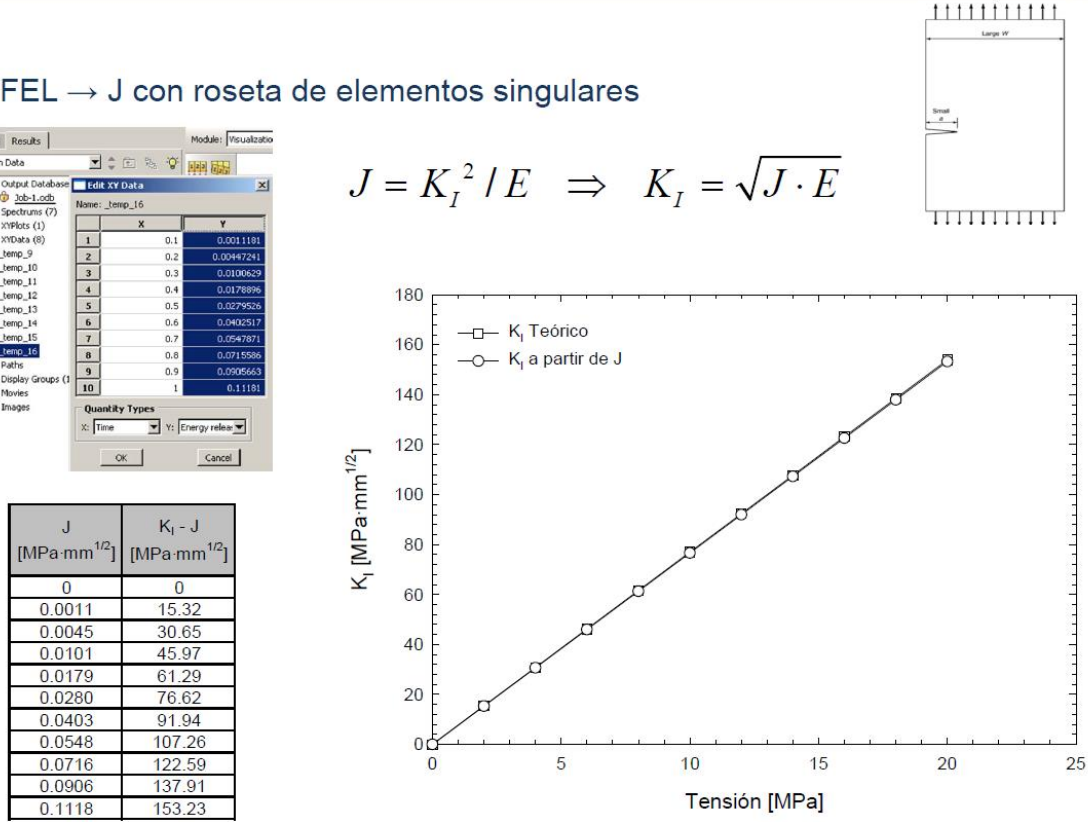

SIMULACIÓN NUMÉRICA DE MATERIALES, COMPONENTES Y ESTRUCTURAS

Fuente: Elaboración propia

2.2 Actuación 2. Uniformización del formato de los objetos didácticos que se utilizarán en el master

La siguiente actuación consistirá en uniformizar el formato, para ello será necesario el consenso de todos los docentes involucrados, ya que de lo contrario las actuaciones descritas en este trabajo no tendrán éxito. En particular, será necesario trabajar sobre el formato de los objetos didácticos generados por los docentes, sobre las plantillas para la entrega de trabajos por parte de los estudiantes y sobre la plantilla para la realización del Trabajo Fin de Master (TFM). De modo que dichos formatos y plantillas sean accesibles desde cada uno de los entornos de trabajo virtual de 
cada universidad. De esta manera tanto docentes como estudiantes involucrados en el Master podrán maximizar la empleabilidad y utilidad de los objetos didácticos.

Para los objetos didácticos ya existentes, generados por los docentes en formato PowerPoint, se ha creado una plantilla para ser aplicada directamente. De este modo, el ejemplo de la Figura 2 pasaría a tener el aspecto que se muestra en la Figura 3.

Figura 3. Ejemplo de objeto didáctico uniformizado.

Máster Interuniversitario: Integridad Estructural y Durabilidad de Materiales, Componentes y Estructuras

Comparación de resultados

- $\mathrm{MFEL} \rightarrow \mathrm{J}$ con roseta de elementos singulares

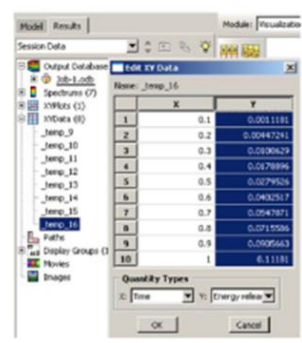

$$
J=K_{I}^{2} / E \Rightarrow K_{I}=\sqrt{J \cdot E}
$$
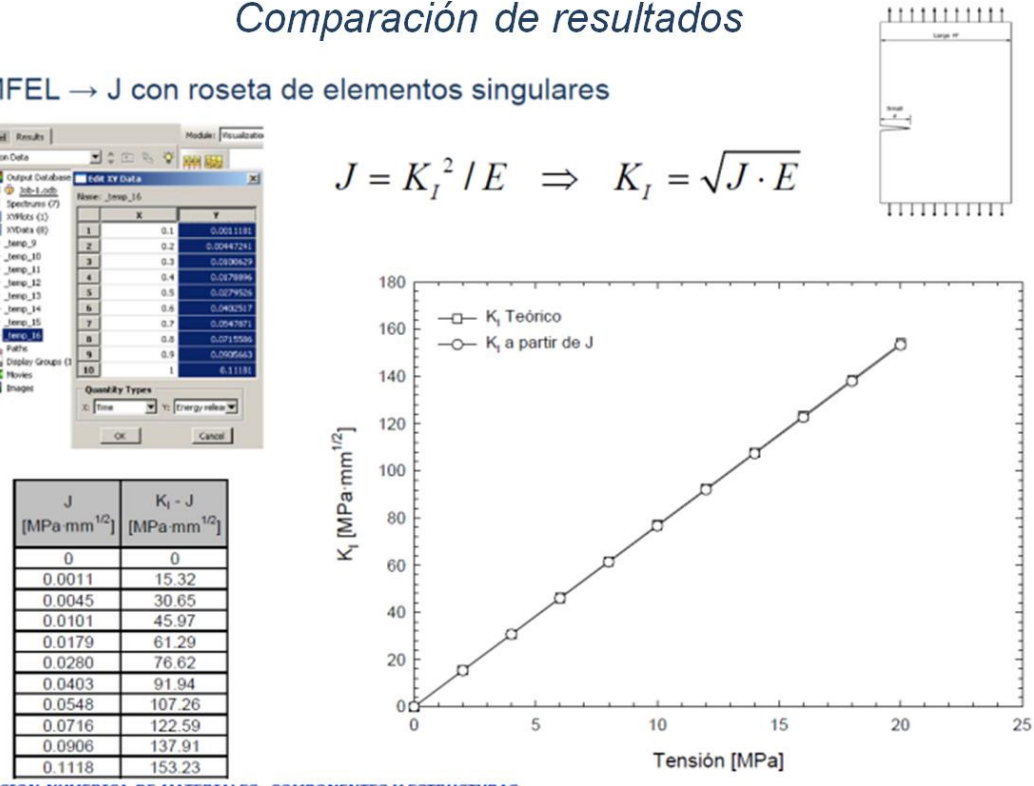

SIMULACION NUMERICA DE MATERIALES, COMPONENTES Y ESTRUCTURAS

Fuente: Elaboración propia

\subsection{Actuación 3. Implantación y seguimiento}

Se ha previsto que las mejoras definidas en las actuaciones anteriores se implanten en el inicio del curso académico, siendo necesario un seguimiento por parte de los coordinadores de cada universidad para su correcta implantación y posible corrección. En el supuesto de que hubiese que tomar medidas correctoras en la uniformización del formato de los objetos didácticos estas se llevarían a cabo a la mayor brevedad posible a lo largo del curso.

Es evidente que el seguimiento del nivel de implantación y la mejora de los objetos didácticos, incluido su formato, en un proceso vivo que debe estar sujeto a una mejora continua, más si cabe cuando se trata de estudios interuniversitarios. De esta manera será posible garantizar una docencia de calidad donde el alumno tendrá acceso a obje- 
tos didácticos de la máxima calidad y de formato uniformizado independientemente de la universidad en la que se matricule del estudio interuniversitario.

\section{Conclusiones}

Las actuaciones planteadas en el presente trabajo sirven de base para lograr una correcta uniformización de formatos de objetos didácticos en estudios interuniversitarios, con el objetivo final de ofrecer al alumnado una mejora continua de los recursos del aprendizaje. De esta manera se conseguirá maximizar la empleabilidad de los objetos didácticos por parte de los estudiantes, haciendo que no afecte el factor campus a los resultados obtenidos por ellos.

\section{Bibliografía}

ÁNGEL SICILIA, M. (2005). "Reusabilidad y reutilización de objetos didácticos: mitos, realidades y posibilidades". En: Revista de Educación a distancia. Disponible en: http://www.um.es/ead/red/M2/sicilia46.pdf

SÁNCHEZ ALONSO, S. (2005). Diseño y uso de objetos didácticos basado en contratos. Tesis Doctoral. Madrid.

WILEY, D. A. (2000). Connecting learning objects to instructional design theory: a definition, a metaphor, and a taxonomy. The Instructional Use of Learning Objects. Ed. D A Wiley. Disponible en: http://reusability.org/read/chapters/wiley. doc

\section{Los autores}

Isidoro Iván Cuesta Segura es Doctor por la Universidad de Burgos y profesor de dicha Universidad.

Jesús Manuel Alegre Calderón es Doctor por la Universidad de Cantabria y profesor de la Universidad de Burgos. 